\title{
Dipeptidyl peptidase IV inhibitor attenuates kidney injury in rat remnant kidney
}

\author{
Kwon Wook Joo ${ }^{1,2+}$, Sejoong Kim ${ }^{3+}$, Shin-young Ahn ${ }^{3}$, Ho Jun Chin ${ }^{2,3}$, Dong-Wan Chae ${ }^{2,3}$, Jeonghwan Lee ${ }^{1}$, \\ Jin Suk Han ${ }^{1,2}$ and Ki Young $\mathrm{Na}^{2,3^{*}}$
}

\begin{abstract}
Background: The inhibition of dipeptidyl peptidase (DPP) IV shows protective effects on tissue injury of the heart, lung, and kidney. Forkhead box O (FoxO) transcriptional factors regulate cellular differentiation, growth, survival, the cell cycle, metabolism, and oxidative stress. The aims of this study were to investigate whether the DPP IV inhibitor sitagliptin could attenuate kidney injury and to evaluate the status of FoxO3a signaling in the rat remnant kidney model.

Methods: Rats were received two-step surgery of 5/6 renal mass reduction and fed on an oral dose of $200 \mathrm{mg} / \mathrm{kg} /$ day sitagliptin for 8 weeks. Before and after the administration of sitagliptin, physiologic parameters were measured. After 8 weeks of treatment, the kidneys were harvested.

Results: The sitagliptin treatment attenuated renal dysfunction. A histological evaluation revealed that glomerulosclerosis and tubulointerstitial injury were significantly decreased by sitagliptin. Sitagliptin decreased DPP IV activity and increased the renal expression of glucagon-like peptide-1 receptor (GLP-1R). The subtotal nephrectomy led to the activation of phosphatidylinositol 3-kinase (PI3K)-Akt and FoxO3a phosphorylation, whereas sitagliptin treatment reversed these changes, resulting in PI3K-Akt pathway inactivation and FoxO3a dephosphorylation. The renal expression of catalase was increased and the phosphorylation of c-Jun N-terminal kinase (JNK) was decreased by sitagliptin. Sitagliptin treatment reduced apoptosis by decreasing cleaved caspase-3 and -9 and Bax levels and decreased macrophage infiltration.

Conclusions: In rat remnant kidneys, DPP IV inhibitor attenuated renal dysfunction and structural damage. A reduction of apoptosis, inflammation and an increase of antioxidant could be suggested as a renoprotective mechanism together with the activation of FoxO3a signaling. Therefore, DPP IV inhibitors might provide a promising approach for treating CKD, but their application in clinical practice remains to be investigated.
\end{abstract}

Keywords: Dipeptidyl peptidase IV, Glucagon-like peptide-1 receptor, FoxO3a, Sitagliptin, Kidney injury

\section{Background}

Glucagon-like peptide-1 (GLP-1) is a gut incretin hormone, whose mimetics have been used as a therapeutic agent for type 2 diabetes. It stimulates pancreatic beta cell proliferation and insulin secretion in a glucose-dependent manner [1]. However, this peptide is almost immediately degraded by dipeptidyl peptidase (DPP) IV in the circulation. DPP IV

\footnotetext{
* Correspondence: kyna@snubh.org

${ }^{\dagger}$ Equal contributors

${ }^{2}$ Department of Internal Medicine, Seoul National University College of Medicine, Seoul, South Korea

${ }^{3}$ Department of Internal Medicine, Seoul National University Bundang Hospital, 166 Gumi-ro, Bundang-gu, Seongnam-si, Gyeonggi-do 463-707, South Korea

Full list of author information is available at the end of the article
}

has a wide variety of substrates that have important roles in cell migration and differentiation, glucose regulation, metabolism, and inflammation [2]. Sitagliptin, a highly selective DPP IV inhibitor, is currently used in the treatment of type 2 diabetes patients to improve glucose tolerance by increasing the half-life of GLP-1 and glucose-dependent insulinotropic peptide (GIP) [3].

The GLP-1 receptor (GLP-1R) agonist exendin-4 has been reported to ameliorate diabetic nephropathy in animals $[4,5]$. Recently, studies have shown that DPP IV inhibitors attenuate kidney injury in diabetic animal models [6,7]. In addition to diabetic nephropathy, DPP IV inhibition protected the kidney against ischemia-reperfusion injury (IRI) [8]. Tissue protective effects of GLP-1 activation

\section{() Biomed Central}


or DPP IV inhibition have also been demonstrated in other organs, including IRI of the lung during transplantation [9-11] and the outcome of myocardial infarction [12,13].

Most cases of chronic kidney disease (CKD) inevitably progress to end-stage renal disease, which has a high associated morbidity and mortality. Although the initiating insult of CKD is variable, the progression of the disease seems to be common to all kidney diseases that involve a vicious cycle of nephron destruction, glomerulosclerosis and tubulointerstitial fibrosis [14]. However, few pharmacologic treatments have been shown to attenuate the progression of CKD. The remnant kidney is a disease model that mimics the progression of CKD in humans. In this model, there is early glomerulosclerosis by week 4 , with segmental sclerosis with tubulointerstitial fibrosis by week 8 . Animals die of uremia starting at week 12 to week 16 [15]. Therefore, 8 weeks after subtotal nephrectomy must be a proper time to observe renal pathology in this model.

Forkhead box $\mathrm{O}$ (FoxO) transcriptional factors regulate various downstream target genes, including those involved in cellular differentiation, growth, survival, the cell cycle, glucose and lipid metabolism, stress, and the detoxification of reactive oxygen species (ROS) [16]. The phosphatidylinositol 3-kinase (PI3K) and serine-threonine kinase Akt/PKB (Akt) pathway regulates FoxO through phosphorylation. The Akt-mediated phosphorylation of FoxO inhibits the activity of FoxO by promoting its interaction with 14-3-3 proteins and its nuclear exportation, and also by inducing its degradation by the proteasome [17]. In the kidney, the FoxO3 transcript is the most abundant among four subfamily members of FoxO proteins [18]. However, there is no study on the status of FoxOs in the remnant kidney model.

Based on the previous reports of tissue protective effects, we hypothesize that DPP IV inhibition could have a positive effect on this animal model of CKD. Due to the diverse regulatory functions of FoxO, our hypothesis is that its signaling might also be modulated by DPP IV inhibition in this model. Therefore, it would be interesting to investigate FoxO signaling in the kidneys. The present study aimed to evaluate whether sitagliptin could attenuate kidney injury in a rat remnant kidney model. Moreover, we investigated the status of FoxO3a signaling after sitagliptin treatment in this model. To do this, we made CKD animal model by two-step surgery of $5 / 6$ renal mass reduction, and then fed these rats on a $200 \mathrm{mg} / \mathrm{kg} /$ day of sitagliptin for 8 weeks.

\section{Methods}

\section{Animal experiments}

All animal procedures were approved by the Institutional Animal Care and Use Committee of the Medical Science Research Institute, Seoul National University Bundang Hospital (BA 1112-095/080-01). Male Sprague-Dawley rats (Orient Bio Inc., Seongnam, South Korea) weighing approximately $200 \mathrm{~g}$ were used. The rats $(n=21)$ were randomly assigned to three groups: sham-operation (sham), subtotal nephrectomy $(\mathrm{Nx})$, and subtotal nephrectomy + sitagliptin treatment $(\mathrm{Nx}+\mathrm{STG})$ groups. After a right subcostal incision, the right kidney was exposed and separated from the adrenal gland under anesthesia with enflurane (Choongwae Pharma Corp., Seoul, South Korea). The lower and upper thirds of the right kidney were resected. After 1 week, the left kidney was removed. The rats of the sham group underwent the same incision and manipulation of the left and the right kidneys without tissue destruction. One week after the second surgical intervention, the rats in the $\mathrm{Nx}+\mathrm{STG}$ group were fed a gelled diet containing $200 \mathrm{mg} / \mathrm{kg} /$ day of sitagliptin (MSD Korea, Seoul, South Korea), and the rats in the sham and $\mathrm{Nx}$ group were fed same gelled diet without sitagliptin. After 8 weeks of treatment, the animals were anesthetized with enflurane, blood samples were obtained, and the kidneys were collected. One portion of the right kidney was fixed in $10 \%$ phosphate-buffered formalin for morphologic and immunohistochemical analyses. The remainder of the right kidney was snap-frozen in liquid nitrogen and stored at $-80^{\circ} \mathrm{C}$ for protein extraction.

\section{Physiologic measurements}

Before and after the administration of a gelled diet with or without sitagliptin, the rats were weighed and placed in metabolic cages, and their urine was collected for $24 \mathrm{~h}$. The urine volume was measured. Serum samples were taken from the tail vein. The blood glucose levels were measured by an Accu-check meter (Roche diagnostics, St Louis, MO, USA). BUN and creatinine levels in the serum and urine were measured using an automatic analyzer (ADVIA 1650, Siemens, Berlin, Germany). Creatinine clearance was calculated and adjusted for body weight.

\section{Determination of DPP IV enzymatic activity}

DPP IV enzymatic activity was assayed in serum using DPP IV Activity Assay Kit (Biovision, Milpitas, CA, USA). A $50 \mu \mathrm{l}$ volume of serum was diluted with $48 \mu \mathrm{l}$ of DPP IV assay buffer and mixed with $2 \mu \mathrm{l}$ substrate Gly-Pro-7Amino-4-Methylcoumarin (AMC) and then incubated at $37^{\circ} \mathrm{C}$ for $30 \mathrm{~min}$. The release of AMC from the substrate was measured with a fluorescence spectrophotometer at $360 \mathrm{~nm}$ of excitation and $460 \mathrm{~nm}$ of emission.

\section{Renal histologic and immunohistochemical analyses}

Tissue for light microscopy and immunoperoxidase staining was fixed in formalin and embedded in paraffin. Three-micrometer sections were stained with hematoxylin and eosin (H\&E). Apoptosis was detected with the enzymatic labeling of DNA strand breaks using terminal deoxynucleotidyl transferase-mediated 
deoxyuridine triphosphate nick end-labeling (TUNEL). TUNEL staining was performed with a Cell Death Detection kit (Roche, Mannheim, Germany). To reveal the total nuclei, the same slides were stained with 4,6'diamidino-2-phenyindole (DAPI) in phosphate-buffered saline. Indirect immunoperoxidase staining with an antiED-1 antibody (Serotec, Oxford, UK) was performed.

\section{Quantification of morphologic data}

All analyses were performed in a blinded manner. Segmental and complete glomerular sclerosis was analyzed using a semiquantitative scoring system from 0 to 4 ( 0 , no glomerulosclerosis; $1,<25 \%$ of glomerular area affected; 2, 25-50\% affected; 3, 50-75\% affected; and 4, 75-100\% affected). At least 30 glomeruli were evaluated under $\times 400$ magnification, and the results were averaged. The tubulointerstitial injury score was estimated based on the number of tubule dilatations, the distortion of the tubular basement membranes, and atrophy from 0 to $3[0$, none $(<5 \%) ; 1$, mild $(5-25 \%) ; 2$, moderate $(25-50 \%)$; and 3 , severe $(>50 \%)]$. More than 10 consecutive fields were examined under $\times 200$ magnification, and the results were averaged. TUNEL (+) apoptotic nuclei were counted in more than 20 consecutive fields under $\times 200$ magnification, and the results were averaged. The mean numbers of infiltrating macrophages (ED-1 positive cells) were calculated by averaging the total numbers of positive cells in more than 20 sequentially-selected, $0.25-\mathrm{mm}^{2}$ grids at $\times 200$ magnification.

\section{Western blot analysis}

Whole kidneys were homogenized in lysis buffer $(250 \mathrm{mM}$ sucrose, $10 \mathrm{mM}$ triethanolamine, $1 \mu \mathrm{g} / \mathrm{ml}$ leupeptin, and $0.1 \mathrm{mg} / \mathrm{ml}$ PMSF titrated to $\mathrm{pH}$ 7.6). The total protein concentration was measured using a bicinchoninic acid protein assay reagent kit (Sigma, St. Louis, MO, USA). The samples were run on SDS-polyacrylamide minigels (Bio-Rad Mini Protean III). The proteins were transferred to nitrocellulose membranes by electroelution. The following proteins were detected using specific antibodies: GLP-1R (Abcam, Cambridge, UK), $\beta$-actin (Santa Cruz Biotech, Santa Cruz, CA, USA), PI3K (BD Bioscience, Franklin Lakes, NJ, USA), total Akt, phospho-Ser473 Akt, total FoxO3a, phospho-Ser253 FoxO3a, total c-Jun N-terminal kinase (JNK), phospho-Thr183/Tyr185 JNK, caspase-3 (all from Cell Signaling Technology, Danvers, MA, USA), caspase-9 (BD Bioscience), Bax (Santa Cruz Biotech), and catalase (Abcam). After incubation with peroxidaseconjugated secondary antibodies (Pierce no. 31458, Rockford, IL, USA), bands were visualized using an enhanced chemiluminescence substrate (ECLTM RPN 2106; Amersham Pharmacia Biotech, Buckinghamshire, UK) before exposure to X-ray film (Hyperfilm; Amersham Pharmacia Biotech, Buckinghamshire, UK). The band densities were quantified by densitometry (GS-700 Imaging Densitometry, Bio-Rad, Hercules, CA, USA).

\section{Statistical analysis}

All of the data are presented as the means \pm S.D. The statistical analyses were performed using SPSS (version 15.0. for Windows; SPSS Inc., Chicago, IL, USA). Difference among three groups were tested with one-way analysis of variance (ANOVA), followed by Turkey's multiple comparison post-test. Statistical significance was indicated by $P<0.05$.

\section{Results}

Table 1 shows the initial and final physiologic parameters of the animals. At the end of the study, rats that received a nephrectomy had a reduced body weight than the shamoperated rats did. However, sitagliptin treatment did not affect body weight or blood glucose levels in the animals. The BUN level remained increased after nephrectomy but was not significantly influenced by treatment with sitagliptin. Serum creatinine levels were increased in the nephrectomized rats, but they were significantly decreased by sitagliptin treatment. Sitagliptin also significantly improved creatinine clearance in nephrectomized rats. Figure 1 shows a representative histological image of the kidneys from each group of animals. In the nephrectomized rats that received sitagliptin, the severity of glomerulosclerosis was significantly reduced compared with the nephrectomized rats that were untreated

Table 1 Physiologic parameters of experimental animals

\begin{tabular}{|c|c|c|c|}
\hline & Sham $(n=6)$ & $N x(n=8)$ & $N x+S T G(n=7$ \\
\hline \multicolumn{4}{|c|}{ Body weight (g) } \\
\hline Initial & $205 \pm 8$ & $204 \pm 9$ & $199 \pm 10$ \\
\hline Final & $563 \pm 34$ & $493 \pm 29^{*}$ & $475 \pm 31^{*}$ \\
\hline \multicolumn{4}{|c|}{ Blood glucose (mg/dl) } \\
\hline Initial & $111 \pm 11$ & $100 \pm 10$ & $111 \pm 6$ \\
\hline Final & $180 \pm 9$ & $184 \pm 21$ & $172 \pm 21$ \\
\hline \multicolumn{4}{|c|}{ BUN (mg/dl) } \\
\hline Initial & $8.27 \pm 2.48$ & $8.63 \pm 1.56$ & $9.94 \pm 3.41$ \\
\hline Final & $12.17 \pm 2.50$ & $28.19 \pm 3.56^{*}$ & $25.04 \pm 7.12^{*}$ \\
\hline \multicolumn{4}{|c|}{$\mathrm{Cr}(\mathrm{mg} / \mathrm{dl})$} \\
\hline Initial & $0.39 \pm 0.08$ & $0.42 \pm 0.06$ & $0.39 \pm 0.11$ \\
\hline Final & $0.52 \pm 0.04$ & $0.90 \pm 0.09^{*}$ & $0.70 \pm 0.19^{* \#}$ \\
\hline \multicolumn{4}{|c|}{$\mathrm{Cl}_{\mathrm{Cr}}(\mathrm{ml} / \mathrm{min} / 100 \mathrm{~g})$} \\
\hline Initial & $0.62 \pm 0.25$ & $0.52 \pm 0.09$ & $0.78 \pm 0.33$ \\
\hline Final & $0.48 \pm 0.05$ & $0.31 \pm 0.03^{*}$ & $0.43 \pm 0.12^{\#}$ \\
\hline
\end{tabular}

The results are expressed as the mean \pm s.d.

*Significantly different with respect to the sham-operated rats; " significantly different with respect to the nephrectomized rats; ${ }^{* \#} P<0.05$.

Sham, sham operation; Nx, nephrectomy; Nx+STG, nephrectomy and sitagliptin treatment.

$\mathrm{Cr}$, creatinine; $\mathrm{Cl}_{\mathrm{Cr}}$, creatinine clearance normalized to body weight. 

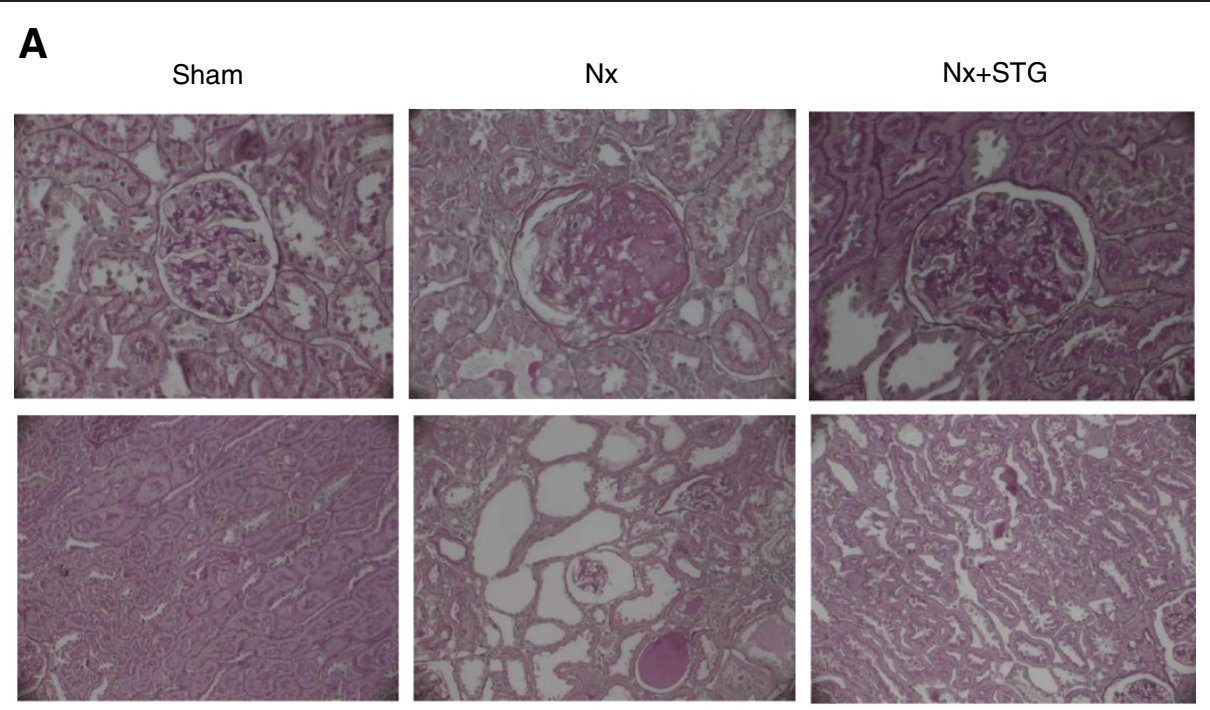

B

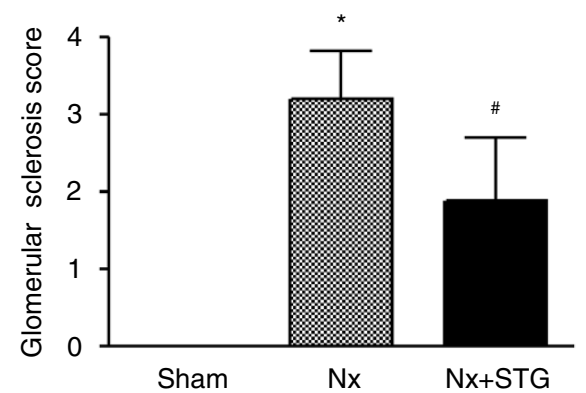

C

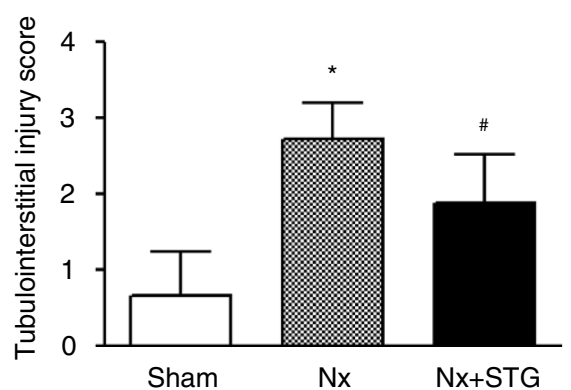

Figure 1 Effects of sitagliptin on renal histology. Representative images of H\&E-stained renal tissues (A). Histological evaluation of glomerular sclerosis (B) and tubulointerstitial injury (C). The extent of glomerular sclerosis and tubulointerstitial injury was evaluated and scored. The results are expressed as the mean \pm s.d. ${ }^{*}$ Significantly different with respect to the sham-operated rats; ${ }^{\#}$ significantly different with respect to the nephrectomized rats; ${ }^{*} P<0.05$. Sham, sham operation; Nx, nephrectomy; Nx+STG, nephrectomy and sitagliptin treatment. Magnification $\times 400$ (upper panel) and x 200 (lower panel).

(score: $1.88 \pm 0.83$ vs. $3.20 \pm 0.63, P<0.05$ ). The degree of tubulointerstitial injury was also significantly reduced by sitagliptin in the nephrectomized rats (score: $1.88 \pm 0.64$ vs. $2.71 \pm 0.49, P<0.05)$. From these results, sitagliptin attenuated renal dysfunction and improved histological damage induced by subtotal nephrectomy.

There was no difference in DPP IV activity in the nephrectomized rats compared with the sham-operated rats (Figure 2A). An almost complete DPP IV inhibition was observed in serum of sitagliptin-treated rats with less than 2\% residual DPP IV activity. The expression of GLP-1R in the kidney was reduced by half after 8 weeks of subtotal nephrectomy (Figure 2B). However, treatment with sitagliptin restored the expression of GLP-1R to the level of sham-operated rats.

We determined the levels of PI3K and phospho-Akt in the kidneys. The levels of PI3K and phospho-Akt in the nephrectomized rats were significantly increased compared to the sham-operated rats, whereas sitagliptin treatment of nephrectomized rats significantly reduced both of these levels (Figure 3). Because PI3K activation and Akt phosphorylation serve as negative regulators of FoxO transcription factors, we next examined the phosphorylation of FoxO3a. Western blotting showed that the phospho-FoxO3a/total FoxO3a ratios were significantly increased in the nephrectomized rats compared to the sham-operated rats (Figure 4). However, the phosphoFoxO3a/total FoxO3a ratios were significantly decreased by sitagliptin treatment. Therefore, sitagliptin restored the inactivation of FoxO3a induced by subtotal nephrectomy.

To investigate whether the status of FoxO3a phosphorylation affected downstream signaling activity, we examined changes in the antioxidant protein catalase. As shown in Figure $5 \mathrm{~A}$, the expression of catalase was significantly increased by sitagliptin treatment. Because JNK is activated by oxidative stress, we next examined JNK phosphorylation. 

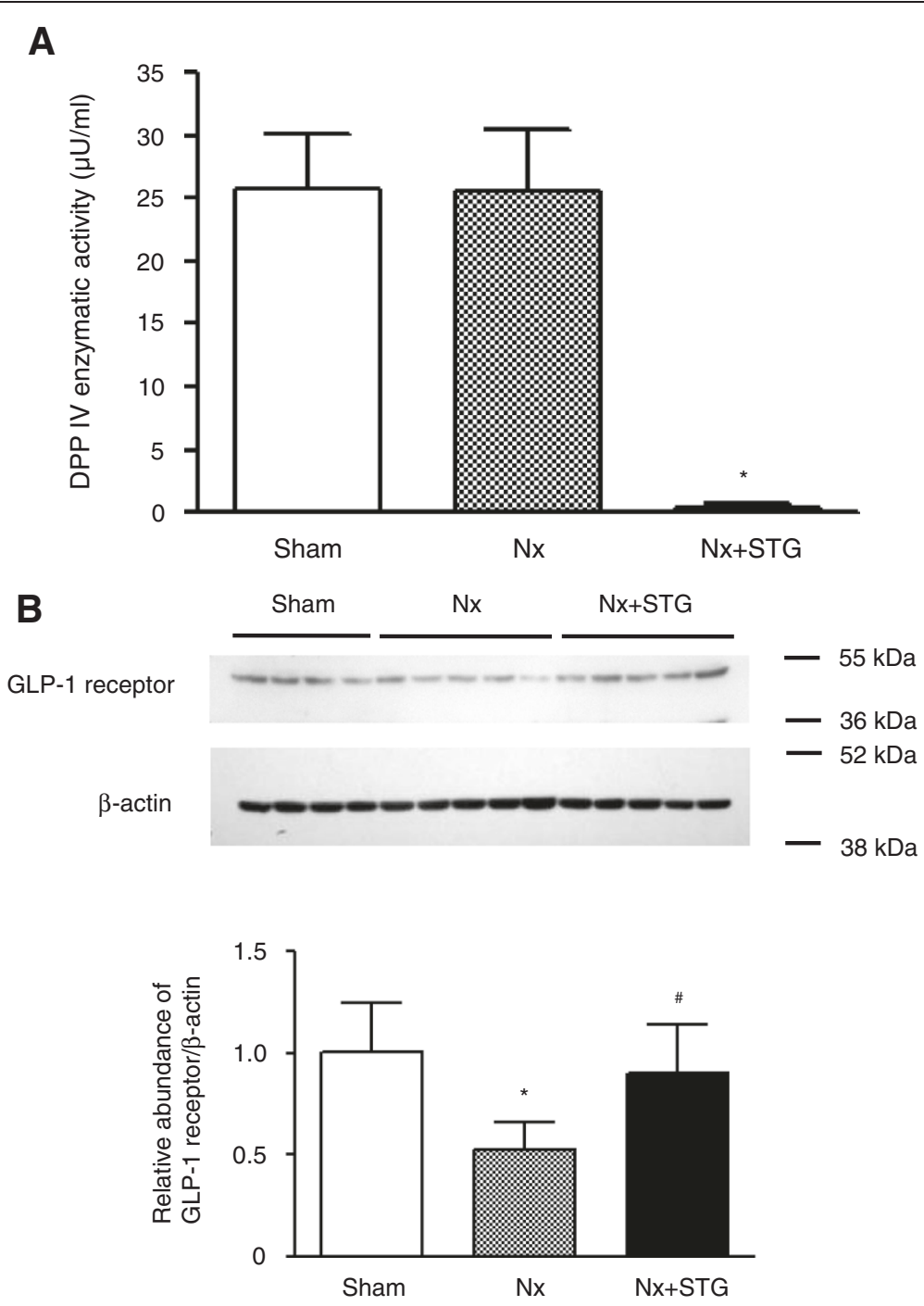

Figure 2 Effects of sitagliptin on DPP IV activity and GLP-1 receptor expression. DPP IV enzymatic activity in serum of rats from each group (A). Representative western blot and group data showing GLP-1 receptor protein abundance in the kidneys of rats from each group (B). The intensity of the bands corresponding to the GLP-1 receptor was corrected by $\beta$-actin levels to obtain relative measures of GLP-1 receptor expression among all samples. The results are expressed as the mean \pm s.d. ${ }^{*}$ Significantly different with respect to the sham-operated rats; " significantly different with respect to the nephrectomized rats; ${ }^{*} P<0.05$. Sham, sham operation; Nx, nephrectomy; Nx+STG, nephrectomy and sitagliptin treatment.

However, there was no difference of the phospho-JNK/total JNK ratios in the nephrectomized rats compared with the sham-operated rats (Figure 5B). The phospho-JNK/total JNK ratios were significantly decreased by sitagliptin treatment. From these results, the antioxidant effect of catalase decreased the activity of JNK in the nephrectomized rats after sitagliptin treatment.

To investigate the extent of apoptosis, we examined kidney sections after detecting DNA fragmentation with an in situ TUNEL assay. Scattered and bright nuclei stained by the TUNEL assay were easily detected in the kidneys of nephrectomized rats, but the number of nuclei was significantly decreased in the kidneys of the sitagliptin-treated rats (Figure 6A). Next, we examined changes in the proapoptotic proteins caspase-3, caspase-9, and Bax by western blot analysis. The cleaved subtypes of both caspase- 3 and caspase-9, and Bax were increased in the kidneys of nephrectomized rats (Figure 6B). However, treatment with sitagliptin significantly reduced the levels of Bax and cleaved subtypes of both caspase- 3 and caspase- 9 in the nephrectomized rats. These results indicate that sitagliptin reduces the extent of apoptosis in the kidneys of nephrectomized rats.

Subtotal nephrectomy was associated with macrophage infiltration in the tubulointerstitium, as determined by an increase in ED-1-positive cells (Figure 7). After counting 


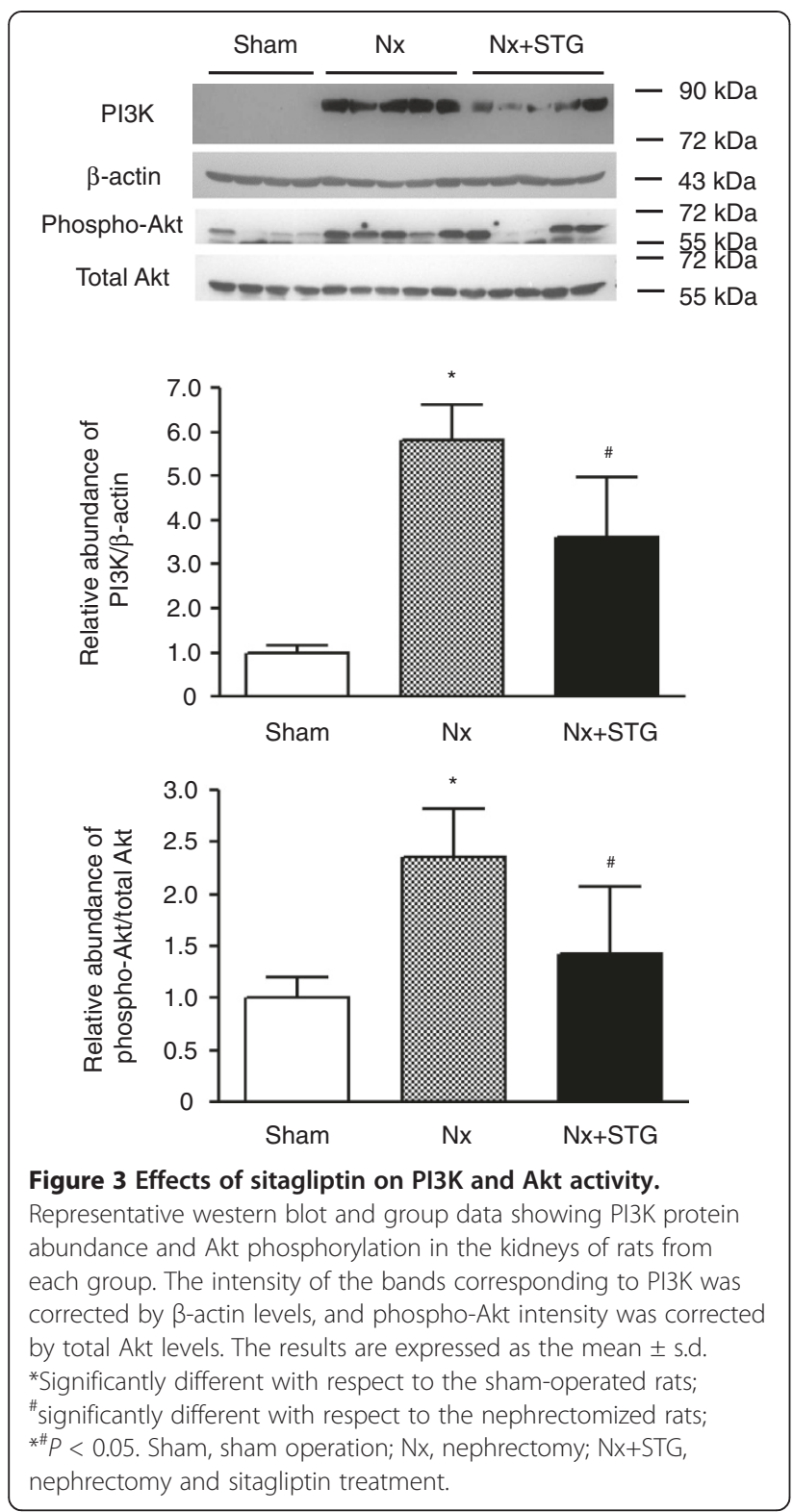

the absolute number of ED-1-positive cells, we observed a marked increase in macrophage infiltration after nephrectomy and a significant reduction in response to sitagliptin treatment. The mean ED-1 score was $94.29 \pm 48.51$ in nephrectomized rats and $34.33 \pm 14.12$ in sitagliptintreated nephrectomized rats.

\section{Discussion}

This study demonstrated that sitagliptin treatment after renal mass reduction showed a renoprotective effect. To the best of our knowledge, this report is the first one to show the effects of sitagliptin, a DPP IV inhibitor, on renal damage in the remnant kidney model. Sitagliptin ameliorated renal dysfunction and attenuated glomerular and tubulointerstitial injury in this model. Treatment
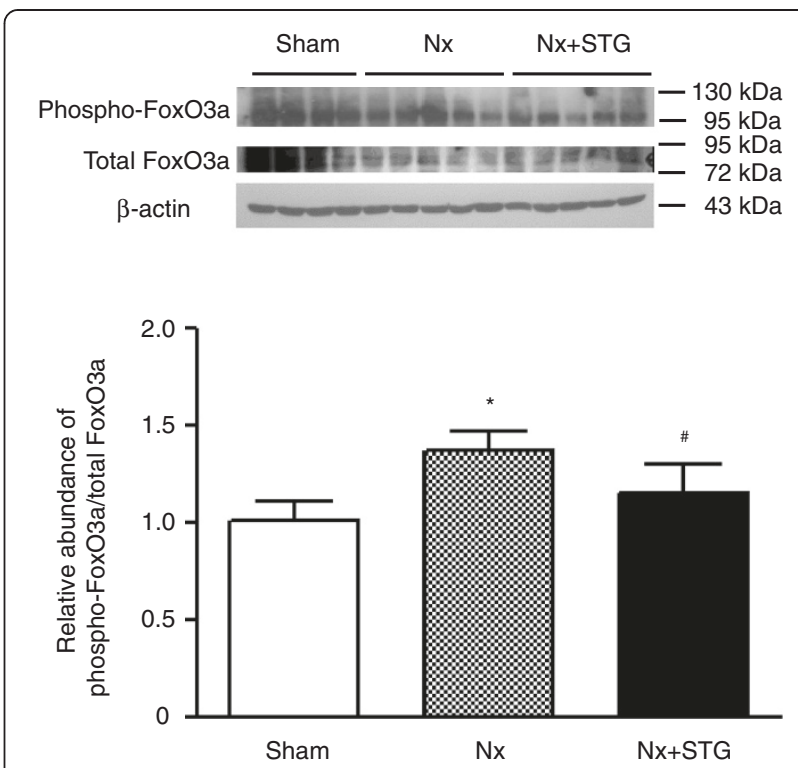

Figure 4 Effects of sitagliptin on FoxO3a phosphorylation. Representative western blot and group data showing FoxO3a phosphorylation in the kidneys of rats from each group. The intensity of the bands corresponding to phospho-FoxO3a was corrected by total FoxO3a levels to obtain relative measures of FoxO3a phosphorylation among all samples. The results are expressed as the mean \pm s.d. ${ }^{*}$ Significantly different with respect to the sham-operated rats; ${ }^{\#}$ significantly different with respect to the nephrectomized rats; ${ }^{*} P<0.05$. Sham, sham operation; $\mathrm{Nx}$,

nephrectomy; Nx+STG, nephrectomy and sitagliptin treatment.

with sitagliptin was found to exert anti-oxidative, anti-apoptotic, and anti-inflammatory effects in this model, together with the inactivation of the PI3K-Akt pathway and the resulting activation of FoxO3a.

In this study, sitagliptin, anti-diabetic drug, did not reduce blood glucose levels in the nephrectomized rats. Therefore, the renoprotective effect of sitagliptin is irrelevant to the reduction of glycemia. DPP IV inhibition does not cause hypoglycemia in a study in healthy male volunteers [19]. Because the action of GLP-1 on insulin secretion is strictly glucose dependent, the risk of hypoglycemia associated with DPP IV inhibitors is low [20].

In the kidney, GLP-1R is strongly expressed in both the glomeruli and proximal tubules $[4,21]$. However, it has been reported that its expression is reduced in diabetic kidneys $[4,7]$. In the heart, the GLP-1R mRNA expression was significantly reduced after subtotal nephrectomy [22]. We first found that GLP-1R expression was markedly decreased in the kidney after subtotal nephrectomy. Judging from the complete inhibition of DPP IV activity in sitagliptin-treated rats, we are sure that sitagliptin significantly raised plasma GLP-1 levels. It has been reported that GLP-1 agonist acts a renoprotective role through increasing GLP-1R expression in diabetic kidneys [4]. Therefore, 

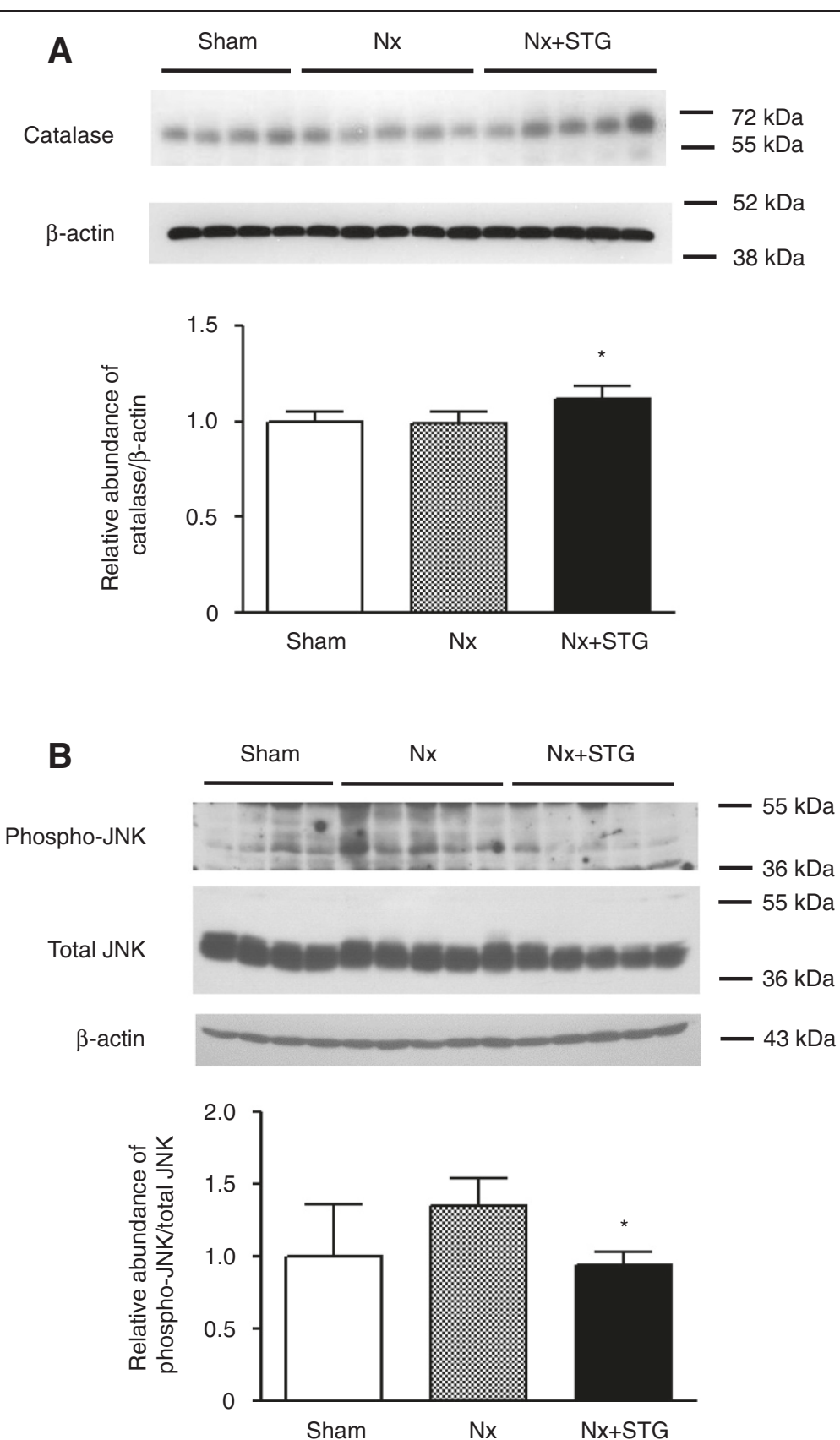

Figure 5 Effects of sitagliptin on oxidative stress. Representative western blot and group data showing catalase expression (A) and JNK phosphorylation (B) in the kidneys of rats from each group. The intensity of the bands corresponding to catalase was corrected by $\beta$-actin levels, and phospho-JNK intensity was corrected by total JNK levels. The results are expressed as the mean \pm s.d. *Significantly different with respect to the nephrectomized rats; ${ }^{*} P<0.05$. Sham, sham operation; Nx, nephrectomy; Nx+STG, nephrectomy and sitagliptin treatment; JNK, c-Jun $\mathrm{N}$-terminal kinase.

chronic sitagliptin treatment in this study might activate renal GLP-1R through DPP IV inhibition because the protein abundance of GLP-1R was significantly increased in kidney homogenates. The dose of sitagliptin used in this study was far above the dose of anti-diabetic usage. To prove the tissue protective effects of DPP IV inhibition, we determined the dose from previous studies $[13,23]$.
Currently, various target genes of FoxOs have been identified in insulin-responsive tissues [24,25]. Therefore, the connection between GLP-1 and FoxO has only been studied in pancreatic beta cells [26]. There are a few studies that have investigated FoxO signaling in the kidney $[27,28]$. Our study is the first to examine the association between GLP-1 and FoxO signaling 

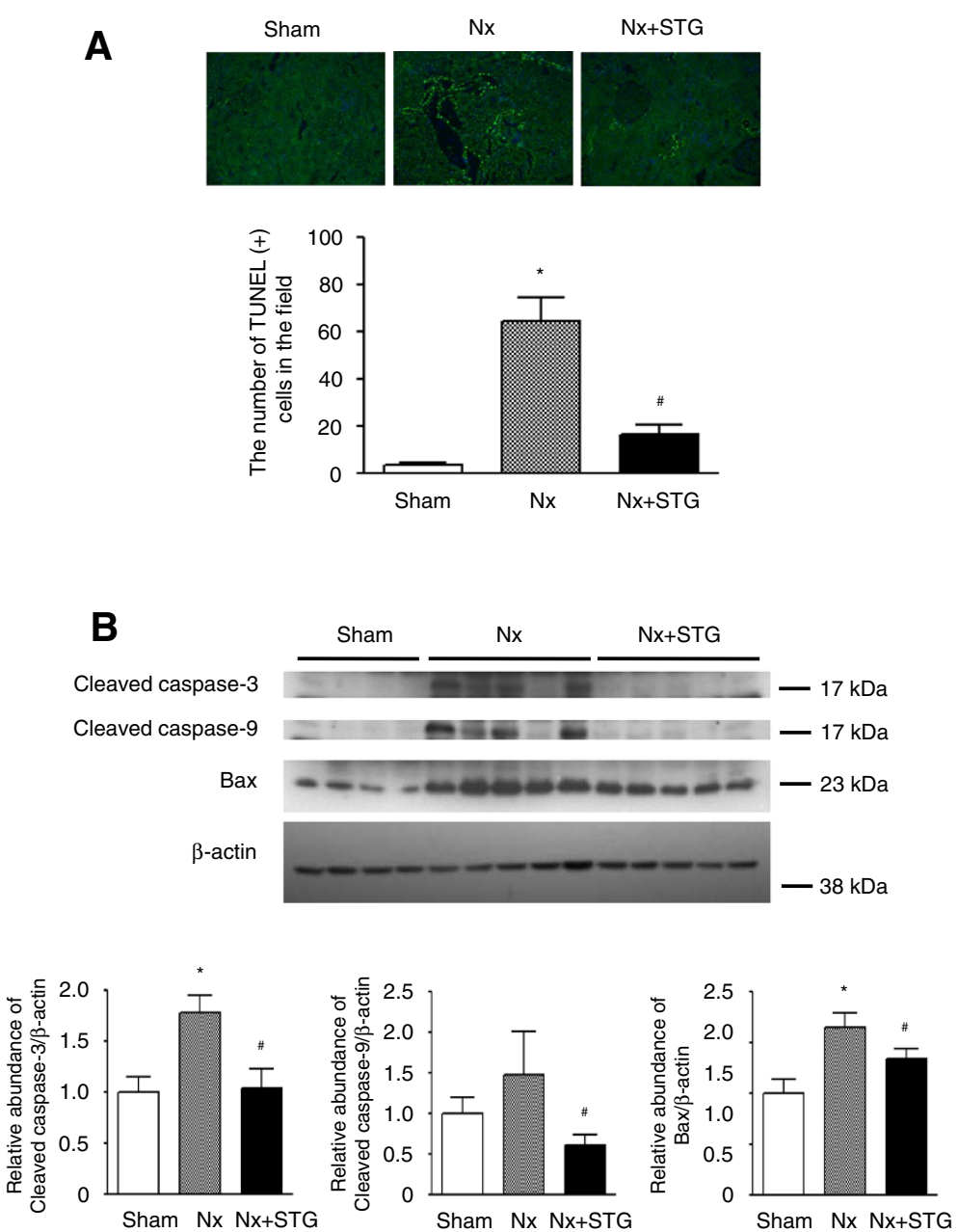

Figure 6 Effects of sitagliptin on apoptosis. Apoptotic cells detected in the kidneys stained with TUNEL (A). Representative western blot and group data showing cleaved caspase-3, cleaved caspase-9, and Bax protein abundance in the kidneys of rats from each group (B). The intensity of the bands corresponding to cleaved caspase-3, cleaved caspase-9, and Bax was corrected by $\beta$-actin levels. The results are expressed as the

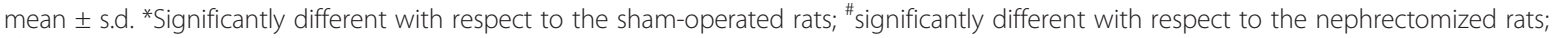
${ }^{*} P<0.05$. Sham, sham operation; Nx, nephrectomy; Nx+STG, nephrectomy and sitagliptin treatment. Magnification $\times 200$.

in rat remnant kidneys. We only investigated the status of FoxO3 in this study because it is the most abundant protein among FoxO subfamily members. The activation of PI3K, the phosphorylation of Akt, and the inactivation of FoxO3a were the main pathway in this disease model. Sitagliptin treatment reversed this pathway.

Although the precise mechanism remains to be elucidated, CKD has been to known to be associated with oxidative stress $[29,30]$. Oxidative stress can occur either as a result of an increased ROS generation, a depressed antioxidant system or both. Catalase is a peroxidase enzyme that is of the major antioxidant defense systems [31]. However, catalase expression and JNK phosphorylation were not changed in this study. Future studies are needed to address these issues. GLP-1R activation using a GLP-1 analog or DPP IV inhibitor reduced oxidative stress in diabetic nephropathy and renal IRI $[4,5,7,8]$. The specific mechanism underlying the anti-oxidative effect of GLP-1R activation remains unclear. In this study, we speculate that the underlying mechanism might be the up-regulation of antioxidant catalase by FoxO3a activation through sitagliptin treatment.

An anti-apoptotic effect mediated by GLP-1R has been suggested in various tissues, including pancreatic beta cells [32], neurons [33], and cardiomyocytes [12]. GLP-1R activation also inhibited apoptosis in diabetic retinopathy [34] and diabetic nephropathy $[4,7]$. The underlying anti-apoptotic mechanism of GLP-1R has been reported in many in vitro studies [35-40]. GLP-1 is capable of inducing downregulation 


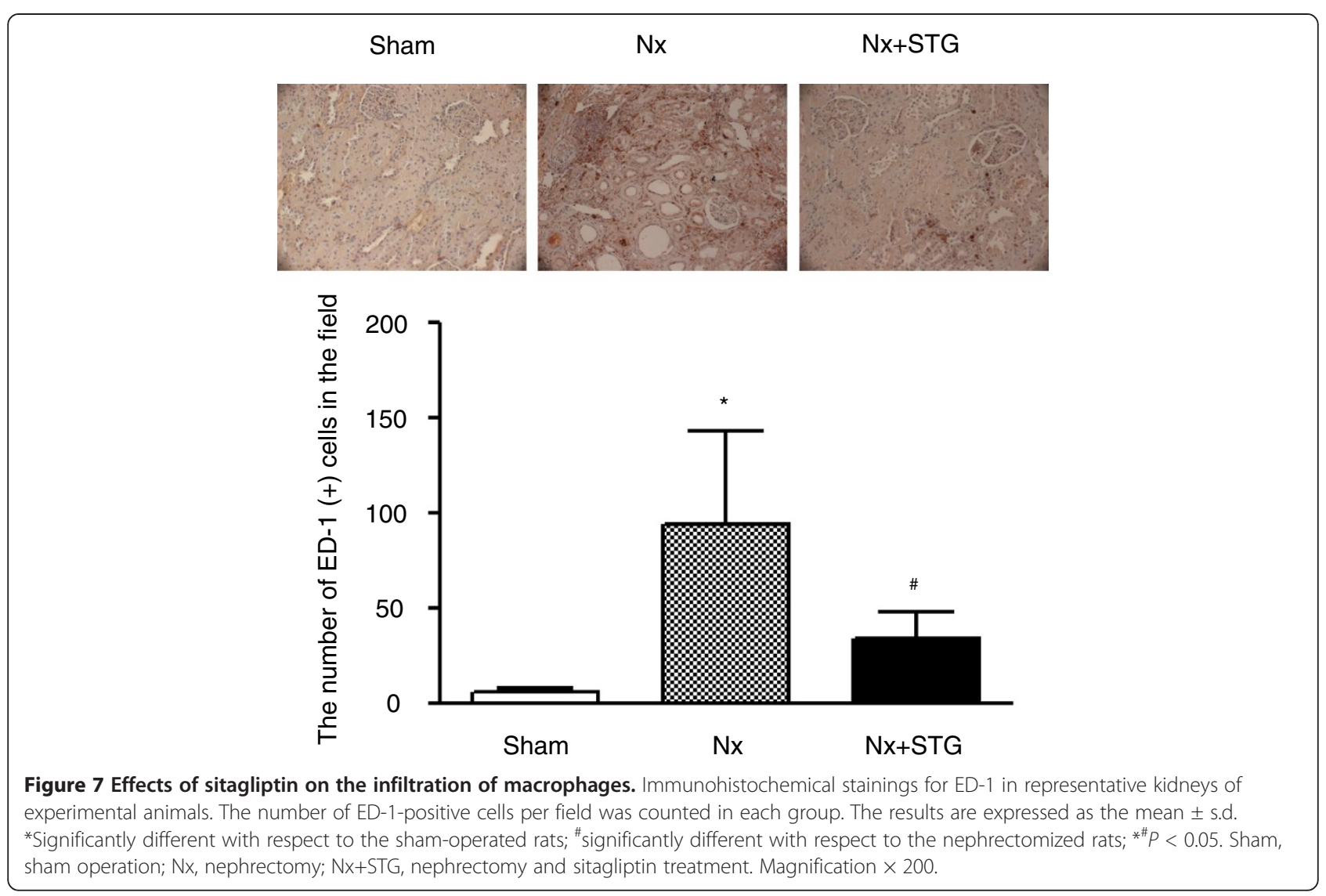

of the pro-apoptotic protein Bax, upregulation of the anti-apoptotic protein Bcl-2, phosphorylation and inactivation of Bad, reducing caspase- 3 activity and DNA fragmentation.

Inflammatory cell infiltration induced by subtotal nephrectomy was attenuated by sitagliptin treatment in this study. A GLP-1R agonist showed anti-inflammatory effects in diabetic nephropathy [4,5]. In kidney IRI, GLP-1R activation using a DPP IV inhibitor ameliorated inflammation [8]. The anti-inflammatory effect of GLP-1R activation was also reported in the animal model of atherosclerosis [41]. Therefore, we speculate that GLP-1R activation by sitagliptin in a CKD animal model showed similar results.

Our study has some limitations. First, we performed the experiments with only three groups of animals without a group of animals with sham-operation and sitagliptin treatment. Due to treatment with a high dose of sitagliptin, we should have included this experimental group to observe any adverse effects in the animals. However, higher doses of sitagliptin than those used in our experiment have been proven to be safe in previous studies $[12,18]$. Moreover, our experiment showed no significant effects on body weight gain or the changes in blood glucose levels in the animals. Second, there is insufficient evidence that the beneficial effect of sitagliptin is through the activation of GLP-1R. DPP IV acts on a wide range of substrates. There is a possibility that other target molecules of DPP IV except GLP-1 may exert the renoprotective effects because plasma GLP-1 levels were not measured in this study. Knockout experiments inhibiting GLP-1 or GLP-1R would be required in the future. Third, there is no direct evidence to determine the causal relationship between GLP-1R and FoxO3a signaling. In vitro experiments using renal cells would also be required to study the direct effects of the GLP-1R on the signaling proteins (PI3K, Akt, JNK, and FoxO3a).

\section{Conclusions}

In summary, sitagliptin treatment attenuated renal dysfunction and structural damage in a model of renal mass reduction. A reduction of apoptosis, inflammation and an increase of antioxidant could be suggested as a renoprotective mechanism, together with the activation of FoxO3a signaling. Therefore, DPP IV inhibitors might provide a promising approach for treating CKD, but their application in clinical practice remains to be investigated. 


\section{Competing interests}

The authors declare that they have no competing interests.

\section{Authors' contributions}

KWJ, JSH, KYN: participated in research design. KWJ, SK, JL: conducted experiments. SA, HJC, DWC: performed data analysis. KYN: wrote or contributed to the writing of the manuscript. All authors read and approved the final manuscript.

\section{Acknowledgements}

The authors thank Yun-Mi Lee and Jin Young Yang for their technical assistance. This study was supported by the Seoul National University Bundang Hospital [Grant 06-2012-091 and 02-2011-013].

\section{Author details}

${ }^{1}$ Department of Internal Medicine, Seoul National University Hospital, Seoul, South Korea. ${ }^{2}$ Department of Internal Medicine, Seoul National University College of Medicine, Seoul, South Korea. ${ }^{3}$ Department of Internal Medicine, Seoul National University Bundang Hospital, 166 Gumi-ro, Bundang-gu, Seongnam-si, Gyeonggi-do 463-707, South Korea.

Received: 26 October 2012 Accepted: 24 April 2013

Published: 27 April 2013

\section{References}

1. Baggio LL, Drucker DJ: Biology of incretins: GLP-1 and GIP. Gastroenterology 2007, 132:2131-2157.

2. Mentlein R: Dipeptidyl-peptidase IV (CD26)-role in the inactivation of regulatory peptides. Regul Pept 1999, 85:9-24

3. Drucker DJ, Nauck MA: The incretin system: glucagon-like peptide-1 receptor agonists and dipeptidyl peptidase-4 inhibitors in type 2 diabetes. Lancet 2006, 368:1696-1705.

4. Park CW, Kim HW, Ko SH, Lim JH, Ryu GR, Chung HW, Han SW, Shin SJ, Bang BK, Breyer MD, Chang YS: Long-term treatment of glucagon-like peptide-1 analog exendin-4 ameliorates diabetic nephropathy through improving metabolic anomalies in db/db mice. J Am Soc Nephrol 2007, 18:1227-1238.

5. Kodera R, Shikata K, Kataoka HU, Takatsuka T, Miyamoto S, Sasaki M, Kajitani N, Nishishita S, Sarai K, Hirota D, Sato C, Ogawa D, Makino H: Glucagon-like peptide-1 receptor agonist ameliorates renal injury through its anti-inflammatory action without lowering blood glucose level in a rat model of type 1 diabetes. Diabetologia 2011, 54:965-978.

6. Mega C, de Lemos ET, Vala H, Fernandes R, Oliveira J, Mascarenhas-Melo F, Teixeira F, Reis F: Diabetic nephropathy amelioration by a low-dose sitagliptin in an animal model of type 2 diabetes (Zucker diabetic fatty rat). Exp Diabetes Res 2011. doi:10.1155/2011/162092

7. Liu WJ, Xie SH, Liu YN, Kim W, Jin HY, Park SK, Shao YM, Park TS: Dipeptidyl peptidase IV inhibitor attenuates kidney injury in streptozotocin-induced diabetic rats. J Pharmacol Exp Ther 2012, 340:248-255.

8. Glorie LL, Verhulst A, Matheeussen V, Baerts L, Magielse J, Hermans N, D'Haese PC, De Meester I, De Beuf A: DPP4 inhibition improves functional outcome after renal ischemia reperfusion injury. Am J Physiol Renal Physiol 2012, 303:F681-F688

9. Lim SB, Rubinstein I, Sadikot RT, Artwohl JE, Önyüksel H: A novel peptide nanomedicine against acute lung injury: GLP-1 in phospholipid micelles. Pharm Res 2011, 28:662-672.

10. Jungraithmayr W, De Meester I, Matheeussen V, Baerts L, Arni S, Weder W: CD26/DPP-4 inhibition recruits regenerative stem cells via stromal cellderived factor- 1 and beneficially influences ischaemia-reperfusion injury in mouse lung transplantation. Eur J Cardiothorac Surg 2012, 41:1166-1173.

11. Zhai W, Jungraithmayr W, De Meester I, Inci I, Augustyns K, Arni S, Hillinger S, Scharpé S, Weder W, Korom S: Primary graft dysfunction in lung transplantation: the role of CD26/dipeptidylpeptidase IV and vasoactive intestinal peptide. Transplantation 2009, 87:1140-1146.

12. Noyan-Ashraf MH, Momen MA, Ban K, Sadi AM, Zhou YQ, Riazi AM, Baggio LL, Henkelman RM, Husain M, Drucker DJ: GLP-1R agonist liraglutide activates cytoprotective pathways and improves outcomes after experimental myocardial infarction in mice. Diabetes 2009, 58:975-983.

13. Sauvé M, Ban K, Momen MA, Zhou YQ, Henkelman RM, Husain M, Drucker DJ: Genetic deletion or pharmacological inhibition of dipeptidyl peptidase-4 improves cardiovascular outcomes after myocardial infarction in mice. Diabetes 2010, 59:1063-1073.

14. Remuzzi G, Bertani T: Pathophysiology of progressive nephropathies. N Engl J Med 1998, 339:1448-1456.

15. Fogo $A B$ : Animal models of FSGS: lessons for pathogenesis and treatment. Semin Nephrol 2003, 23:161-171.

16. Zhang $X$, Tang N, Hadden TJ, Rishi AK: Akt, FoxO and regulation of apoptosis. Biochim Biophys Acta 1813, 2011:1978-1986.

17. Tzivion G, Dobson M, Ramakrishnan G: FoxO transcription factors; Regulation by AKT and 14-3-3 proteins. Biochim Biophys Acta 1813 2011:1938-1945

18. Jacobs FM, van der Heide LP, Wijchers PJ: FoxO6, a novel member of the FoxO class of transcription factors with distinct shuttling dynamics. J Biol Chem 2003, 278:35959-35967.

19. Bergman AJ, Stevens C, Zhou Y, Yi B, Laethem M, De Smet M, Snyder K, Hilliard D, Tanaka W, Zeng W, Tanen M, Wang AQ, Chen L, Winchell G, Davies MJ, Ramael S, Wagner JA, Herman GA: Pharmacokinetic and pharmacodynamic properties of multiple oral doses of sitagliptin, a dipeptidyl peptidase-IV inhibitor: a double-blind, randomized, placebocontrolled study in healthy male volunteers. Clin Ther 2006, 28:55-72.

20. Weir GC, Mojsov S, Hendrick GK, Habener JF: Glucagonlike peptide I (7-37) actions on endocrine pancreas. Diabetes 1989, 38:338-342.

21. Schlatter $P$, Beglinger C, Drewe J, Gutmann H: Glucagon-like peptide 1 receptor expression in primary porcine proximal tubular cells. Regul Pept 2007, 141:120-128.

22. Chaykovska L, von Websky K, Rahnenführer J, Alter M, Heiden S, Fuchs H, Runge F, Klein T, Hocher B: Effects of DPP-4 inhibitors on the heart in a rat model of uremic cardiomyopathy. PLoS One 2011, 6:e27861.

23. Lim S, Choi SH, Shin H, Cho BJ, Park HS, Ahn BY, Kang SM, Yoon JW, Jang HC, Kim YB, Park KS: Effect of a dipeptidyl peptidase-IV inhibitor, des-fluoro-sitagliptin, on neointimal formation after balloon injury in rats. PLoS One 2012, 7:e35007.

24. Kim JJ, Li P, Huntley J, Chang JP, Arden KC, Olefsky JM: FoxO1 haploinsufficiency protects against high-fat diet-induced insulin resistance with enhanced peroxisome proliferator-activated receptor gamma activation in adipose tissue. Diabetes 2009, 58:1275-1282.

25. Fang CX, Dong F, Thomas DP, Ma H, He L, Ren J: Hypertrophic cardiomyopathy in high-fat diet-induced obesity: role of suppression of forkhead transcription factor and atrophy gene transcription. Am J Physiol Heart Circ Physiol 2008, 295:H1206-H1215.

26. Kim W, Egan JM: The role of incretins in glucose homeostasis and diabetes treatment. Pharmacol Rev 2008, 60:470-512.

27. Percy CJ, Brown L, Power DA, Johnson DW, Gobe GC: Obesity and hypertension have differing oxidant handling molecular pathways in age-related chronic kidney disease. Mech Ageing Dev 2009, 130:129-138.

28. Chung HW, Lim JH, Kim MY, Shin SJ, Chung S, Choi BS, Kim HW, Kim YS, Park CW, Chang YS: High-fat diet-induced renal cell apoptosis and oxidative stress in spontaneously hypertensive rat are ameliorated by fenofibrate through the PPARa-FoxO3a-PGC-1a pathway. Nephrol Dial Transplant 2012, 27:2213-2225.

29. Vaziri ND, Oveisi F, Ding Y: Role of increased oxygen free radical activity in the pathogenesis of uremic hypertension. Kidney Int 1998, 53:1748-1754

30. Miyata T, Kurokawa K, Van Ypersele De Strihou C: Advanced glycation and lipoxidation end products: role of reactive carbonyl compounds generated during carbohydrate and lipid metabolism. J Am Soc Nephrol 2000, 11:1744-1752.

31. Fridovich I: Superoxide radical and superoxide dismutases. Annu Rev Biochem 1995, 64:97-112.

32. Li Y, Hansotia T, Yusta B, Ris F, Halban PA, Drucker DJ: Glucagon-like peptide-1 receptor signaling modulates beta cell apoptosis. $J$ Biol Chem 2003, 278:471-478

33. During MJ, Cao L, Zuzga DS, Francis JS, Fitzsimons HL, Jiao X, Bland RJ, Klugmann M, Banks WA, Drucker DJ, Haile CN: Glucagon-like peptide-1 receptor is involved in learning and neuroprotection. Nat Med 2003, 9:1173-1179.

34. Zhang Y, Wang Q, Zhang J, Lei X, Xu GT, Ye W: Protection of exendin-4 analogue in early experimental diabetic retinopathy. Graefes Arch Clin Exp Ophthalmol 2009, 247:699-706.

35. Marzioni M, Alpini G, Saccomanno S, Candelaresi C, Venter J, Rychlicki C, Fava G, Francis H, Trozzi L, Benedetti A: Exendin-4, a glucagon-like peptide 
1 receptor agonist, protects cholangiocytes from apoptosis. Gut 2009, 58:990-997.

36. Qin Z, Sun Z, Huang J, Hu Y, Wu Z, Mei B: Mutated recombinant human glucagon-like peptide-1 protects $\mathrm{SH}-\mathrm{SY} 5 \mathrm{Y}$ cells from apoptosis induced by amyloid-beta peptide (1-42). Neurosci Lett 2008, 444:217-221.

37. Liu JH, Yin F, Guo LX, Deng XH, Hu YH: Neuroprotection of geniposide against hydrogen peroxide induced PC12 cells injury: involvement of PI3 kinase signal pathway. Acta Pharmacol Sin 2009, 30:159-165.

38. Kimura R, Okouchi M, Fujioka H, Ichiyanagi A, Ryuge F, Mizuno T, Imaeda $K$, Okayama N, Kamiya Y, Asai K, Joh T: Glucagon-like peptide-1 (GLP-1) protects against methylglyoxal-induced $\mathrm{PC} 12$ cell apoptosis through the $\mathrm{PI3K} / \mathrm{Akt} / \mathrm{mTOR} / \mathrm{GCL} /$ redox signaling pathway. Neuroscience 2009, 162:1212-1219.

39. Quoyer J, Longuet C, Broca C, Linck N, Costes S, Varin E, Bockaert J, Bertrand G, Dalle S: GLP-1 mediates antiapoptotic effect by phosphorylating Bad through a beta-arrestin 1-mediated ERK1/2 activation in pancreatic betacells. J Biol Chem 2010, 285:1989-2002.

40. Ravassa S, Zudaire A, Carr RD, Díez J: Antiapoptotic effects of GLP-1 in murine HL-1 cardiomyocytes. Am J Physiol Heart Circ Physiol 2011, 300:H1361-H1372.

41. Arakawa M, Mita T, Azuma K, Ebato C, Goto H, Nomiyama T, Fujitani Y, Hirose T, Kawamori R, Watada H: Inhibition of monocyte adhesion to endothelial cells and attenuation of atherosclerotic lesion by a glucagon-like peptide-1 receptor agonist, exendin-4. Diabetes 2010, 59:1030-1037.

doi:10.1186/1471-2369-14-98

Cite this article as: Joo et al.: Dipeptidyl peptidase IV inhibitor attenuates kidney injury in rat remnant kidney. BMC Nephrology 2013 14:98.

\section{Submit your next manuscript to BioMed Central and take full advantage of:}

- Convenient online submission

- Thorough peer review

- No space constraints or color figure charges

- Immediate publication on acceptance

- Inclusion in PubMed, CAS, Scopus and Google Scholar

- Research which is freely available for redistribution 